\title{
Source Digital Image Identification based on Cross-correlation
}

\author{
Yong-jie XIE, Yi BAO, Sen-feng TONG, Yu-hao YANG \\ College of Electrical Engineering, Zhengzhou University \\ Zhengzhou, China \\ zzu_na@163.com
}

\begin{abstract}
Source camera detecting, which is about establishing whether or not the images of interest are taken by the same camera, is a challenging problem. Due to solve the digital image source identification problems, a new method is proposed using Cross-correlation Analysis. In the digital camera imaging process, PRNU (Photo-Response-NonUniformity) can be introduced as identify specific digital camera's fingerprint. The average operator of image residual noise collection product by images is to use mostly for the digital camera reference pattern noise model obtain. In order to locate the position of the sharp peak correlation value, crosscorrelation measure reference $P R N U$ with image residual noise in the frequency domain is used. The position information considered as recognition feature of the image to which it yield. The experimental results show that the method had significantly improved average rate of source digital image identification.
\end{abstract}

Keywords-Source camera identification; PRNU; Crosscorrelation

\section{INTRODUCTION}

With the rapid development of science and technology, digital camera is widely used in all areas of life, such as media out-lets, businesses, industries, and even court of law as a primary way to present, process, and store information. It is also possible to edit and manipulate digital information with very low cost, effort, and expertise. The availability of such technologies and their ease of use, risk the credibility of digital information we use in our daily lives. Therefore, the question of verifying their integrity and origin is rapidly increasing on importance. Due to source camera identification technology research is more and more popular, the 'Dresden Image Database' for Benchmarking Digital Image Forensics has been made available for forensic investigators[1]. The images in the experiments are come from it. Typical examples for acquired scenes are depicted in Fig. 1.

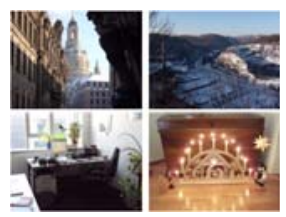

Fig. 1 Examples of indoor and outdoor scenes-natural and man-made-in the 'Dresden Image Database'.

Different brands of digital camera use different sensor and especially the use of camera image processing technology different. Therefore, even if they used the same scene shooting, conditions under the same style (exposure, date, and time, etc.), the output of image is also exist slight differences. Source identification is according to these differences to trace which camera takes the image. At the heart of digital camera is the image sensor. It is an array of rows and columns of photodiode elements, or pixels. When light strikes the pixel array, they can convert the light intensity to the voltage of the proportion of the photoelectric value each corresponding to a pixel. Most digital cameras use CCD (charge-coupled device) sensor, and some also use CMOS (complementary metal oxide semiconductor) sensor. As show in Fig. 2, the two main components of pattern noise are fixed pattern noise and photo-response non-uniformity noise. The FPN is caused by some pixels in a sensor will collect more charge than others because of small differences in their geometry or their electrical properties. It is additive noise and can be suppressed automatically by subtracting a dark frame from every image they take. PRNU is more robust to most processing so that, usually different methods use PRNU for camera identification. In [2], a novel and effective approach for the camera identification has been proposed. This approach identifies the digital image source according to the PRNU noise that is a unique stochastic characteristic of image sensors. They first put forward by using the fingerprint of noise (such as CCD or CMOS model of noise) to identify digital camera. An important issue for the reliable identification is how to extract just the PRNU noise from the contaminated noises on each image. In order to relax the effects of scene content, the method proposed in [3] enhances the contaminated noise so that the strong components are attenuated and the weak components are amplified. Through the analysis of the noise of residual keep PRNU noise strong regional and discard mode noise weak area [4]. The experiment showed that improve recognition rate. Dirik A.E. et al. [5] proposed through the image change in the intensity of the form and shape features dust discrimination model of digital image source. Sensor dust pattern produces dusts pecks on the image, which is not changed unless the sensor surface is cleaned. But need to point out that because of the ordinary consumer level a nonSLR digital camera does not need to replace lens, this method can be aimed at digital SLR cameras source identification only. In order to reduce costs, most consumer digital cameras just use one image sensor. As both CCD and CMOS sensors can only sense luminance, color filtering and demosaicing are the common steps in digital still cameras (DSC) to cost-effectively produce color. In color filtering, a color filter array (CFA) is used to remove the unwanted colors so that specific colors can be measured at each pixel. Different digital camera manufacturers use color filter matrix 
and interpolation algorithm (demosaicing algorithm) different. Through the estimated CFA interpolation rule to identify different camera model, the method achieved an average accuracy of $97.7 \%$ for 10 -camera classification [6]. But, for the same digital camera makers use the same color filter matrix and the CFA interpolation algorithm.

The rest of paper is organized as follows. Section 2 presents the traditional design ideas and the procedure followed to acquire the set of benchmark images, where we also describe the process for extracting the reference PRNU for each camera and the correlation detector. A new source camera identification model is introduced in Section 2.2. Section 3 experiments show it have high recognition rate.

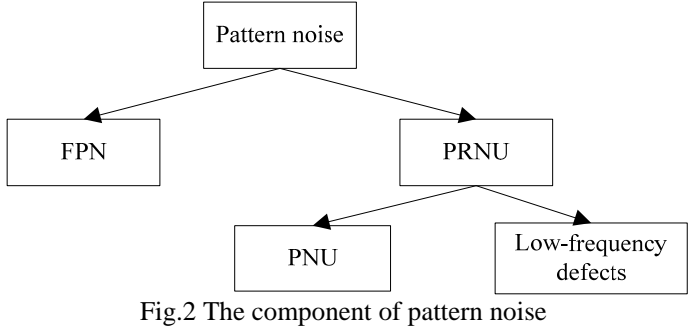

II. MODEL AND ALGORITHM

\section{A. Traditional Model}

Recently, the photo-response non-uniformity (PRNU) was proposed as a sensor fingerprint, an equivalent of biometric for imaging sensors. The PRNU is turn up in all semiconductor imaging sensors, including CCD and CMOS architectures. It is caused by the inconsistency and the uniqueness of manufacturing imperfections and sensitivity to light that even sensors made from the same silicon wafer manufacturer would possess uncorrelated PRNU noise pattern, which can be extracted from the images produced by the sensors. It is almost no major change in time and can be reliably detected in virtually all images even after lossy compression or additional processing. These properties make PRNU a robust fingerprint for identifying the origin and verifying the integrity of images. The PRNU is produced by add a set of the image residual noises which images take by one digital camera. Since PRNU is mainly a high-frequency signal, so a traditional model for extracting it from image is:

$$
n=I-F(I)
$$

where $F$ is a low pass denoising filter which filters out the PRNU noise pattern. $\mathrm{n}$ is image residual noise which contains most PRNU. Although various denoising filters can be used as F, the wavelet-based denoising process is the best perform of it. The filter described in [7] has been tested as effective in producing good results.

Most camera brands and models have a similar imaging pipeline. Because of CCD\&CMOS imaging sensors are color-blind, each pixel captures only intensity information. Real images have $R \& G \& B$ color channel and each channel can be calculated respectively image residual noise. To do that, it has three the same dimension image residual noises which are $n_{r}, n_{g}$ and $n_{b}$. Single color channel of image residual noise can be obtained by:

$$
w_{c i}=\frac{\sum_{k=1}^{L} n^{i}{ }_{k}}{L}
$$

where $n_{k}{ }^{i}$ is the $k t h$ image and the corresponding PRNU of single color channel $i$ extracted from digital camera $c$ is $w_{c i}$. Note the multiplication operation in Eq. (2) is element-wise. In Fig. 3, the algorithm schematic diagram is shown.

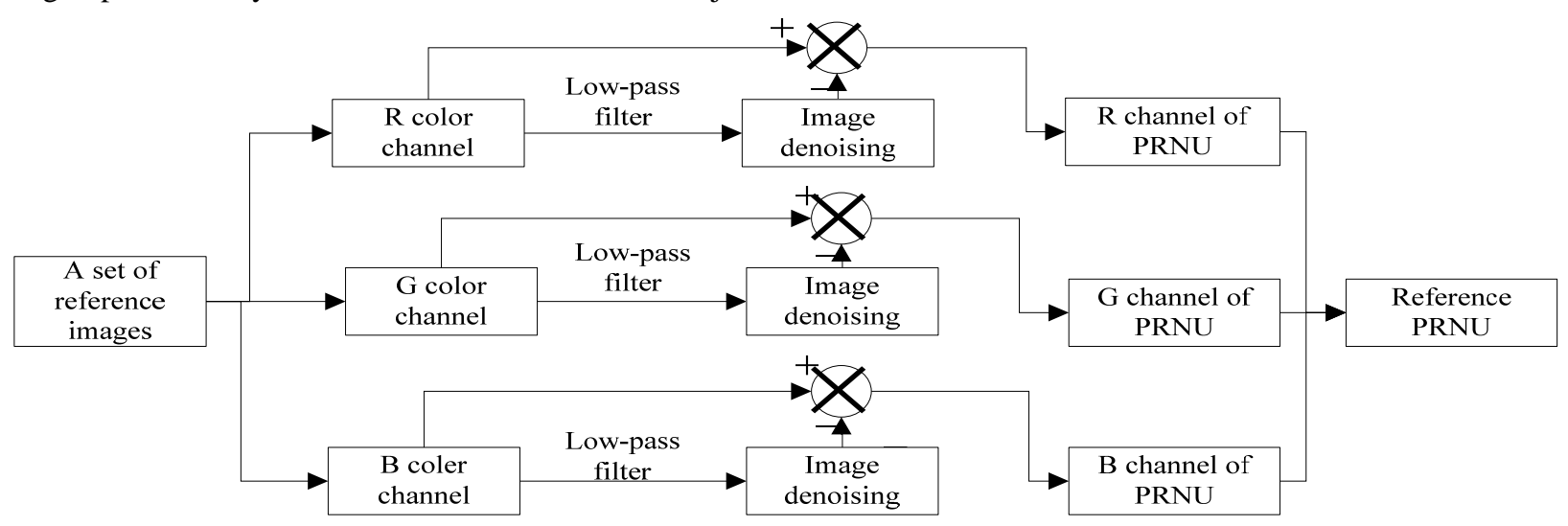

Fig.3 Reference PRNU extraction process

For the convenience of calculation, the PRNU of single color channel transforms into a reference PRNU model, a model coefficients are the RGB images convert to grayscale images as the same use in [8].

$$
w_{c}=0.3 * w_{c r}+0.6 * w_{c g}+0.1 * w_{c b}
$$

Everywhere in traditional methods, the detection scheme

is made using correlation-based comparison. The normalized correlation detector is:

$$
\operatorname{corr}\left(w_{c}, n_{t}\right)=\frac{\left(w_{c}-\bar{w}_{c}\right) \cdot\left(w_{c}-\bar{n}_{t}\right)}{\left\|w_{c}-\bar{w}_{c}\right\|\left\|w_{c}-\bar{n}_{t}\right\|}
$$

where ( $\overline{)}$ denotes mean of it. 


\section{B. New Detection Model}

Source camera detecting, which is about establishing whether or not the images o $\mathrm{f}$ interest are taken by the same camera, is a challenging problem. Different from the current version of the proposed method, the detection model dropped up normalized correlation detector. We selected as an alternative test statistics the following normalized cross-correlation easily calculable using DFT. Finally, a locator located the peak position $\left(x_{0}, y_{0}\right)$.

As an example, we describe how to calculate the reference PRNU, $w_{c}$, from $n$ a set of reference images captured by a specific camera and how to use our method. Step 1: Separate the three color channels $I^{i}, i \in\{R, G, B\}$ of a color image $I$. Choose an adaptive denoising filter. Perform denoising operation on each color channel of reference images $I_{r j}^{i} j=1,2 \ldots n$ and test images $I_{t k}^{i}$, $k=1,2 \ldots m$.

Step 2: Subtract each color channel of the denoised image from its corresponding source channel to get the residual image $n^{i}$.

Step 3: Calculate PRNU $w_{c i}, i \in\{R, G, B\}$ of each color channel and reference PRNU $w_{c}$.

Step 4: The cross-correlation coefficient $c_{w n}$ between the reference PRNU and the noise residual $n_{t}$ is calculated as:

$$
\begin{gathered}
c_{w n}(j, k)=\sum_{x, y} w_{c}(x, y) n_{t}{ }^{*}(x-j, y-k) \\
=\sum_{u, v} W_{c}(u, v) N_{t}^{*}(u, v) \exp \left[i 2 \pi\left(\frac{u j}{M}+\frac{v k}{N}\right)\right] \\
j=1 . . M, k=1 \ldots N
\end{gathered}
$$

complex conjugation; uppercase letters represent the DFT of their lowercase counterparts, as given by the relation

$$
W_{c}(u, v)=\sum_{x, y} \frac{W_{c}(x, y)}{\sqrt{M N}} \exp \left[-i 2 \pi\left(\frac{u x}{M}+\frac{v y}{N}\right)\right]
$$

Step 5: Finally, take position of the location of the maximum peak and calculate it to origin $\mathrm{O}(0,0)$ Euclidean distance $\rho(x 0, y 0)>T h$ as the source of the judgment standard:

$$
\begin{aligned}
& m\left(x_{0}, y_{0}\right)=\max \left\|c_{r n}(j, k)\right\| \\
& j=1 \ldots M, k=1 \ldots N \\
& \rho\left(x_{0}, y_{0}\right)=\sqrt{x_{0}{ }^{2}+y_{0}{ }^{2}}>T h
\end{aligned}
$$

where $T h$ is a threshold selected to obtain a desired false positive rate.

\section{EXPERIMENTAL}

To verify the performances of the presented new detection model, we have carried out identification tests on 1190 photos of natural scenes taken by seven cameras, each reference set responsible for 50 and test set responsible for 120 . In order to reduce the amount of calculation, experiment of the photos taken only $512 \times 512$ center pixels. Digital camera parameters are shown in Table 1. It is conformable to use the actual situation in life, each image contents should be rich as far as possible, including, landscape, building, indoor and outdoor, different illumination condition, etc.

where $\mathrm{N}$ and $\mathrm{M}$ are the image dimensions; $(*)$ denotes

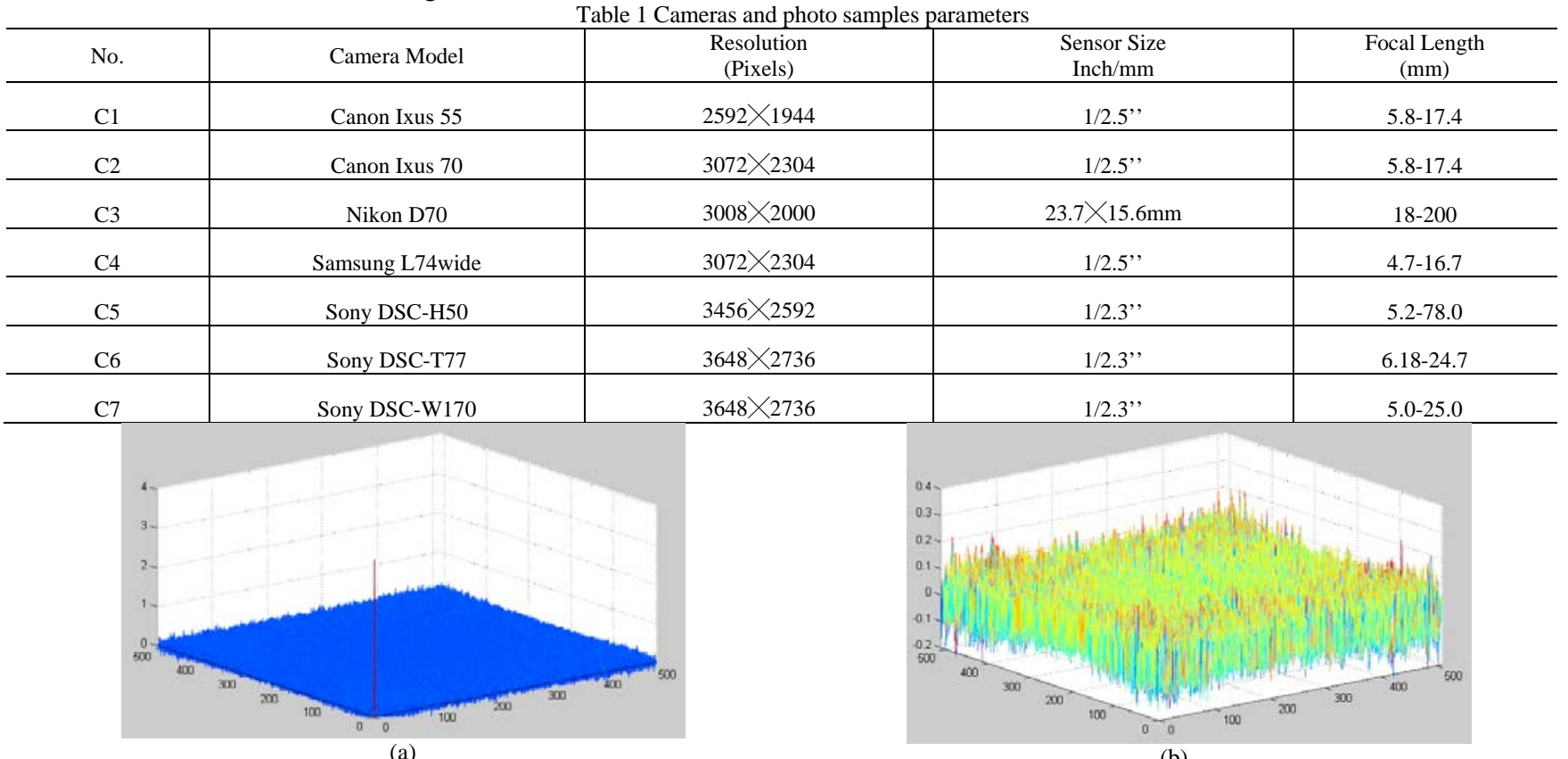

(a)

Fig. 4 The cross-correlation values calculate with the $\mathrm{C} 1$ reference PRNU . (a) A test image of C1 and reach peak at $(0,0)$. (b) A test image of C2 and reach peak at $(92,498)$. 


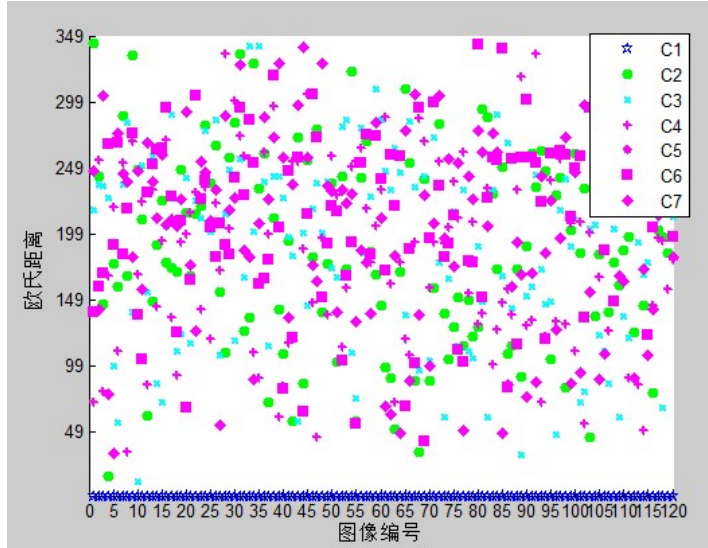

Fig. 5 Euclidean distance distribution of seven reference PRNU and test photos of C1

Table 2 Identification accuracy of the new model

\begin{tabular}{l|l|l|l|l|l|l|l}
\multicolumn{8}{c}{ Table 2 Identification accuracy of the new model } \\
\hline No. & C1 & C2 & C3 & C4 & C5 & C6 & C7 \\
\hline Recognition (\%) & 100 & 100 & 94.16 & 93.33 & 86.66 & 100 & 100 \\
\hline
\end{tabular}

\section{CONCLUSIONS}

We present a method that can be used to verify whether digital images were obtained using the certain digital camera. It is based on detecting a digital camera output reference PRNU in the noise residuals of both images. With the frequency domain cross-correlation analysis method, it true locates coefficient peak position. We use this feature information to identify seven different digital cameras for source identification and average recognition accuracy rate is up to $96.3 \%$.

\section{ACKNOWLEDGMENT}

This work was supported in part by Provincial Major Scientific and Technological Research Projects Grant Nos. 092101210100.
From the Fig.4 and Fig.5, the test photos of C1 cameras and the reference PRNU cross-correlation values in near origin of coordinates $O(0,0)$ reach peak, and other camera photos in the far origin of coordinates $O(0,0)$ reach peak. In this paper is the cross correlation coefficient peak position feature can be effective identification picture source camera. The experiments of each digital camera have excellent recognition accuracy rate which has shown in Table 2.

1] Gloe T. ,Böhme R. The 'Dresden image database' for benchmarking digital image forensics[C]. Proceedings of the ACM Symposium on Applied Computing, 2010:1584-1590.

[2] Lukas J., Fridrich J.,Goljan M. Digital camera identification from sensor pattern noise [J]. IEEE Transactions on Information Forensics and Security.2006,1(2): 205- 214.

[3] Li Chang-Tsun. Source Camera Identification Using Enhanced Sensor Pattern Noise [J]. IEEE Transactions on Information Forensics and Security, 2010,5(2): 280-287.

[4] Liu Bei-Bei, Hu Yong-jian, Lee Heung-Kyu. Source camera identification from significant noise residual regions[C]. Proceedings - International Conference on Image Processing, 2010: 1749-1752.

[5] Dirik A.E.,Sencar H. T., Memon N. Source Camera Identification Based on Sensor Dust Characteristics[J]. IEEE Transactions on Information Forensics and Security, 2008,3(3): 539-552.

[6] Cao Hong, Kot A.C. Accurate Detection of Demosaicing Regularity for Digital Image Forensics[J]. IEEE Transactions on Information Forensics and Security.2009,4(4): 899-910.

[7] Portilla J., Strela V., Wainwright M.J.,Simoncelli E.P. Image denoising using scale mixtures of Gaussians in the wavelet domain[J]. IEEE Transactions on Image Processing. 2003,12(11) :1338- 1351.

[8] Fridrich J. Digital Image Forensic Using Sensor Noise[J].IEEE Signal Processing Magazine. 2009,26(2):26-37. 